Aim The project aimed to improve awareness and knowledge of end of life care needs for people with LD; increase confidence and skills of staff providing end of life care for people with LD.

Methods A partnership group met to make a funding bid; development and delivery of an education project across sectors and professionals, successfully submitted to HEE regionally. Held consensus curriculum development workshops to inform the training programme. Literature review and evaluation commissioned from university (report due September 2018). A twoday training programme with support materials, e.g. resource book. Programme delivered by utilising both specialist LD and end of life care knowledge and practice. $6 \times$ two-day courses were delivered between October 2017 and April 2018.

Results The delivery of the project has been beset with personnel issues such as staff sickness and changes in roles which was a challenge for project management. Training day evaluations were positive about the learning, presentation and gaining knowledge for practice. Practitioners provided examples of learning use in practice as feedback illustrating their increased confidence. Collaboration between specialist LD practitioners and the hospice Education Team has been a positive experience. Conclusions De-brief project meeting recommended further development of advance care planning and skills and confidence in end of life care for people with LD locally. A multiprofessional attendance was achieved. Collaborative hospice/LD team working contributed to the overall experience.

\section{P-39 INCLUDING PEOPLE WITH LEARNING DISABILITIES IN END OF LIFE CARE}

${ }^{1}$ Monica Reardon, ${ }^{2}$ Michael Banner. 'Marie Curie, Cardiff, UK; ${ }^{2}$ Marie Curie, Penarth, UK

\subsection{6/bmjspcare-2018-hospiceabs.64}

Background The CIPOLD (Heslop, Blair, Fleming, et al., 2013) study found that people with learning disabilities (PWLD) died on average 13 years (men) or 20 years (women) sooner than the average UK population. Despite $57 \%$ of studied deaths being 'expected', only 20\% received support from specialist palliative care services (Heslop, Blair, Fleming et al., 2013). Initial consultations with PWLD, their families, and professionals in Cardiff and the Vale found limited knowledge of palliative care services and this lack of awareness limited access to services.

Aims The Big Lottery funded Improving Access project aimed to identify barriers to PWLD accessing palliative care services and implement activities to reduce these.

Methods 12 organisations and 54 individuals consulted, including PWLD, families/carers, health and social care professionals. Project advisory group includes a PWLD and inclusion of PWLD was key to the project's success.

Results Consultations identified the following needs:

- Improved community engagement and education to increase awareness of services.

- Opportunities for PWLD to be supported to talk about end of life

- Varied formats of accessible information.

Project delivering the following activities:

- Education and workshops to professionals, PWLD, and carers on topics including bereavement and advance care planning.

Audiences included learning disability teams, attendees of the
Learning Disability Wales annual conference, and bereavement teams.

- Promoting Marie Curie Easy Read materials.

- Working with PWLD to develop 'what to expect' videos that can be used to educate PWLD about hospices and palliative care.

145 people reached through face-to-face events.

Conclusion The project has increased internal staff awareness of the challenges PWLD face in accessing services so that these needs can be considered in shaping and delivering services. It has improved awareness of palliative care services for PWLD, carers, and professionals and created opportunities for PWLD to talk about end of life. Involvement of PWLD is vital in reaching more people and ensuring the voices of PWLD are heard and included.

\section{P-40 ATTITUDES TOWARDS LESBIAN, GAY, BISEXUAL AND TRANSGENDER (LGBT) FAMILIES: SURVEY METHODOLOGY}

${ }^{1,2}$ Catrin Evans. 'Open University, London, UK; ${ }^{2}$ Volunteer, Young Adults' Group, St Christopher's Hospice, London, UK

\subsection{6/bmjspcare-2018-hospiceabs. 65}

Background Families and patients accessing hospice care in the UK are diverse. LGBT families are now possible following social change, blended families, and medical advancements. Herek's (1984) Attitudes towards Lesbians and Gay Men was an influential survey. Existing studies are predominantly qualitative. Survey research by Frias-Navarro et al. (2009) and Habarth (2015) shaped the literature, giving rationale for this study.

Research question Do homophobic, traditional, and authoritarian views and beliefs predict attitudes towards LGBT families? The two-part hypothesis was; Humans with (i) prejudiced authoritarian, heteronormative views and (ii) traditional viewpoints relating to gender, will not view LGBT families positively.

Method This survey methodology drew on three established scales by Herek (1984), Habarth (2015), and Frias-Navarro et al. (2009). After piloting, participants were recruited through Open University colleagues, social media, professional and personal networks. 104 adult participants were recruited aged between 20 and 76. Multiple regression analysis took place.

Results Results supported the two-part hypothesis.

Humans who held i) prejudiced, authoritarian, heteronormative, and ii) gender-traditional views, will not view LGBT families positively. The proportion of variance that can be explained by the model is $\mathrm{R} 2=0.82$. The results indicated that the model was a significant predictor $F(2,95)=101.1, p=0.001$ Both predictor variables contributed significants $(p=0.01)$ $\beta 1=0.225 \beta 2=0.237$.

Conclusion The results link with existing literature. While Herek's (1984) research was situated in the 1980s HIV/AIDS crisis, its findings of traditional LGBT prejudice are still relevant. Habarth (2015) finding subtler heteronormativity is also important. Awareness of this may have relevance for hospice care in terms of how we approach diverse families and care for those who self-identify as LGBT. There has been limited research in the UK to date. Scope for future research includes mixed method design and sampling a wider-ranging gender and sexual diversity. 